\title{
O Desenvolvimento da Confiança nas Comunidades Terapêuticas e o seu Impacto na Adesão ao Tratamento
}

\author{
The Development of Trust in Therapeutic Communities \\ and its Impact on Treatment Adherence
}

\author{
Luís A. Machado* \& Ana Veloso \\ ${ }^{a}$ Escola de Psicologia, Universidade do Minho, Braga, Portugal
}

\begin{abstract}
Resumo
A confiança é reconhecida como um elemento essencial para o estabelecimento da relação terapêutica, com um importante impacto na adesão ao tratamento. No entanto, existem poucos estudos e modelos teóricos que permitam a sua conceptualização e mensuração neste contexto. A presente investigação tem como objectivo estudar o desenvolvimento da confiança nas Comunidades Terapêuticas e o seu impacto na adesão ao tratamento. Foi adoptada uma metodologia mista e participaram nesta investigação, 231 sujeitos distribuídos por nove Comunidades Terapêuticas. Os resultados mostram a existência de dois níveis de confiança (na relação dos residentes com os técnicos e com as instituições) e apresentam-na como um elemento fundamental para o desenvolvimento da relação terapêutica e para a adesão ao tratamento. Palavras-chave: Comunidades Terapêuticas; Adesão ao Tratamento; Confiança nos Técnicos; Confiança nas Instituições.
\end{abstract}

\begin{abstract}
Trust is recognized as an essential element for the establishment of therapeutic relationship and has a major impact on treatment adherence. However, there are few studies and theoretical models which allow its conceptualization and measurement within this context. This research aims to study the development of trust in Therapeutic Communities and its impact on treatment adherence. It was adopted a mixed methodology and 231 subjects spread over nine Therapeutic Communities participated of the study. The results show the existence of two levels of trust (with the technical staff and with the institutions) and present it as a key element for developing therapeutic relationship and treatment adherence.

Keywords: Therapeutic Communities; Treatment Adherence; Trust in Technical Staff; Trust in the Institutions.
\end{abstract}

Apesar dos estudos sobre a confiança contarem já com algumas décadas e abrangerem várias áreas de interesse, na área das dependências e mais especificamente nas Comunidades Terapêuticas (CT’s) para a recuperação de toxicodependentes, são poucos os estudos que procuram desenvolver e aprofundar modelos teóricos que permitam a sua conceptualização e avaliação. Por esta razão, houve a necessidade de criar um modelo de desenvolvimento da confiança para este contexto que, como veremos posteriormente, foi concebido a partir da análise da literatura existente nas várias áreas de investigação.

Vários autores salientam que a confiança na relação terapêutica é a base do sucesso do tratamento nas CT’s (Bell, 1994; De Leon, 1994; Soyez, Tatrai, Broekaert, \& Bracke, 2004), enquanto a falta de confiança é apresentada como uma das causas de abandono (Bell, 1994). A

* Endereço para correspondência: l-machado@hotmail.com e alveloso@psi.uminho.pt confiança é frequentemente relacionada com melhor integração dos residentes (Soyez et al., 2004), maior abertura para falar de problemas sensíveis, maior tolerância à dor e à frustração, seguimento das orientações dadas, cumprimento das exigências de funcionamento (Bell, 1994), continuidade no tratamento e mudança de comportamentos (Bell, 1994; De Leon, 1994). Segundo Dugan, Trachtenberg e Hall (2005), em situações de elevada vulnerabilidade como no campo das dependências, a existência de confiança é fundamental para o sucesso da relação terapêutica. Leite e Vasconcellos (2003) apoiamse nos resultados de diversos estudos, para destacar a confiança como um factor decisivo para a adesão ao tratamento.

O estudo da confiança, neste contexto, ganha maior relevância tendo em conta as características pessoais desta população e do próprio método de tratamento. Embora por norma cada residente tenha um técnico responsável pelo acompanhamento do seu processo, a estrutura e organização das CT's obriga a que todos os residentes se 
relacionem com quase todos os técnicos, criando múltiplas interacções (Bell, 1994). As CT's diferem de outras abordagens de tratamento, principalmente no uso do conceito de comunidade, onde os próprios residentes são os principais agentes da mudança (Vieira, 2007). Os residentes das CT's são caracterizados como pessoas com níveis reduzidos de confiança e defensivos (Bell, 1994), com défices de competências sociais e dificuldade em confiar naqueles que representam autoridade (Pickens \& Fletcher, 1991). Vários autores referem que os consumidores de drogas são pessoas com dificuldade na adesão a tratamentos (Lima, 2006; Scaduto \& Barbieri, 2009; World Health Organization [WHO], 2003), sendo referenciadas, em alguns estudos, taxas de abandono durante o primeiro mês de $50 \%$ (Scaduto \& Barbieri, 2009). Entre os factores que dificultam a adesão encontram-se o baixo nível sócio-económico, comorbidades psiquiátricas, falta de suporte familiar ou social (O’Brien \& McLellan, 1996), considerar que o problema não é grave o suficiente para merecer tratamento, considerar o tratamento não relevante para promover mudanças de vida, medo do estigma por procurar tratamento (Fontanella \& Turato, 2002) e o estilo de vida caótico dos consumidores de drogas (Colombrini, Lopes, \& Figueiredo, 2006). Também os problemas que decorrem da relação com os prestadores de cuidados como a percepção, por parte dos consumidores, de incompetência, crueldade e distanciamento dos profissionais, têm sido referenciados como causadores de dificuldades na adesão (Fontanella \& Turato, 2002).

O principal ponto de discórdia, impeditivo de uma definição consensual da adesão, assenta no papel atribuído a quem procura os cuidados. Enquanto alguns autores atribuem um papel passivo e de obediência ao prestador de cuidados de saúde, outros conferem-lhe um papel activo e participativo (Leite \& Vasconcellos, 2003; Lima, 2006; Scaduto \& Barbieri 2009). O conceito de adesão adoptado neste estudo enquadra-se na definição da Organização Mundial de Saúde, que a define como "a medida em que o comportamento de uma pessoa corresponde às recomendações de um prestador de cuidados de saúde" (WHO, 2003). Assim como a confiança, a adesão resulta de uma escolha de quem procura os cuidados de saúde. De facto, de acordo com os modelos de adesão e confiança adoptados neste estudo, estes conceitos têm vários pontos em comum: dependem de uma decisão pessoal (aderir vs não aderir; confiar vs não confiar), que é influenciada pelas características dos vários intervenientes e do contexto e são frequentemente referidas (a confiança na relação terapêutica e a adesão aos tratamentos prescritos) pela sua associação com resultados mais favoráveis. A dificuldade de adesão apontada aos consumidores de drogas e a importância atribuída à confiança como um factor decisivo para que a mesma se verifique, reforça a necessidade do seu estudo no contexto das CT's.

Apesar de alguma literatura apresentar uma perspectiva unidimensional da confiança (Costa, 2004), a pers- pectiva vigente apresenta-a como um processo relacional, multidimensional, dinâmico e complexo. Ao nível das definições encontradas na literatura, verifica-se que a vulnerabilidade, a percepção de risco, a predisposição para confiar, as expectativas positivas e a natureza relacional, são aspectos frequentemente relacionados directa ou indirectamente com a confiança (Rosseau, Sitkin, Burt, \& Camerer, 1998). A partir da análise da literatura, foi realizada uma síntese que integra os cinco aspectos anteriormente mencionados e define a confiança como um estado psicológico que assenta na intenção de aceitar a vulnerabilidade ou risco percebido, baseada na expectativa positiva acerca das intenções e comportamentos da parte em quem a confiança é depositada.

Relativamente aos factores que moderam o desenvolvimento da confiança, existe uma grande dispersão de variáveis estudadas, sendo nesta categoria que a área de estudo em que os autores estão inseridos assume um peso mais relevante. Para este estudo, foi adoptado o modelo de Dietz e Hartog (2006), no qual as variáveis moderadoras da confiança são distribuídas por três categorias: (a) características de quem confia; (b) características da parte em quem é depositada a confiança; (c) características contextuais da relação.

Nas características de quem confia encontram-se a "propensão para confiar", o estado de "vulnerabilidade" e a "percepção do risco existente" decorrente do mesmo (Dugan et al., 2005; Goudge \& Gilson, 2005; Mayer, Davis, \& Schoorman, 1995). Os sujeitos variam na sua "propensão para confiar" (que depende da educação, das experiências de vida e da cultura), sendo que esta influencia a sua decisão de confiar (Bigley \& Pearce, 1998; Dietz \& Hartog, 2006; Mayer et al., 1995). A "vulnerabilidade" representa a possibilidade da pessoa que confia vir a sofrer perdas ou outras experiências negativas resultantes do comportamento da parte em quem a confiança é depositada (Tzafrir \& Dolan, 2004). Os indivíduos que confiam colocam-se de alguma forma em situação de vulnerabilidade em relação a outros com quem interagem em situações sociais, relacionamentos pessoais ou sistemas (Bigley \& Pearce, 1998). A "percepção de risco", resultante do estado de vulnerabilidade, anteriormente descrito, assenta na incerteza dos motivos, intenções e acções de outros de quem se depende e representa a possibilidade de danos para a pessoa que confia (Goudge \& Gilson, 2005).

No campo das características da parte em quem é depositada a confiança, fez-se um trabalho de análise e síntese das variáveis estudadas nas várias áreas de investigação, que resultou na decisão de adoptar o modelo de Mayer et al. (1995). Este modelo sustenta como variáveis moderadoras da confiança a "competência”, a "integridade" e a "benevolência". A "competência” integra os conhecimentos e as capacidades necessárias para responder às necessidades de quem confia, competências de comunicação e a capacidade para desenvolver a relação terapêutica. A “integridade” remete para percepção de que 
a pessoa em quem se confia defende valores e princípios considerados aceitáveis para quem confia. Aqui podem ser incluídos a honestidade, o respeito, a justiça, a coerência, a fidelidade e a garantia de que a informação resultante do comportamento de confiança não será utilizada para provocar dano na pessoa que confia. Finalmente a "benevolência" significa que a parte em quem se confia não se vai aproveitar da vulnerabilidade de quem confia. Pode ser entendida como uma demonstração de preocupação genuína, de boa fé e interesse pelo outro.

Relativamente às características contextuais da relação, Rosseau et al. (1998) referem que o contexto é essencial para a compreensão da confiança. A percepção e interpretação da parte que confia acerca do contexto onde está inserido o relacionamento, afecta tanto a necessidade de confiar como a avaliação da confiabilidade da outra parte (Mayer et al., 1995). Uma característica que pode ser encontrada nesta categoria é a "natureza da relação" - se é uma relação forçada ou de livre opção. Num estudo realizado com consumidores de drogas verificou-se que aqueles que tiveram possibilidade de escolher o prestador de serviços de saúde manifestaram níveis mais elevados de confiança (Ostertag, Wright, Broadhead, \& Altice, 2006). O "tipo de ligação" existente entre as partes também se enquadra nas características contextuais - se a relação resulta de uma parceria profissional, de uma relação médico doente, de um contexto social, ou outra (Goudge \& Gilson, 2005). Outra característica contextual prende-se com o "nível de conhecimento" que quem confia tem sobre a parte em quem deposita a confiança - a possibilidade de prever o comportamento depende da existência de conhecimento prévio das características da parte em quem é depositada a confiança (Bigley \& Pearce, 1998; Mcallister, 1995). Por último, no que toca às características contextuais, destaca-se o papel do "tempo" no desenvolvimento da confiança - se é uma relação curta ou de longa duração e se existem encontros esporádicos ou frequentes. A percepção que quem confia tem acerca das características da parte em quem deposita a confiança aumenta ao longo do tempo e do desenvolvimento da relação (Goudge \& Gilson, 2005; Schoorman, Mayer, \& Davis, 2007).

A decisão de confiar tem sido discutida na literatura a partir de dois princípios que são apresentados como complementares: baseada em processos cognitivos e/ou baseada em aspectos afectivos (Goudge \& Gilson, 2005; Mcallister, 1995). Schoorman et al. (2007) salientam que as emoções influenciam a percepção dos antecedentes e a própria confiança, principalmente no início da relação. Estes autores também referem que apesar do efeito das emoções se dissipar com o tempo, os aspectos cognitivos permanecem.

Seguindo este raciocínio, pode-se concluir que a decisão de confiar baseada em emoções acontece principalmente no início de uma relação de confiança, quando não existe conhecimento acerca da confiabilidade da parte em quem a confiança vai ser depositada. Por outro lado, os aspectos cognitivos ganham maior peso com o desenvolvimento da relação de confiança ao longo do tempo e com o conhecimento das características que permitem julgar a confiabilidade.

Dietz e Hartog (2006) defendem que o processo de desenvolvimento da confiança se completa através de uma sequência composta por três etapas: (1) Confiança como uma crença - do acesso possível de uma parte (A) à confiabilidade da outra parte (B) resulta uma crença de que "B" se vai comportar de acordo com os interesses de "A"; (2) Confiança como uma decisão - além da crença na confiabilidade de "B", tem que existir a decisão de "A" se tornar vulnerável a "B"; (3) Confiança como uma acção - para " $A$ " demonstrar realmente confiança em "B", este tem que (além da crença na confiabilidade e da decisão de se tornar vulnerável) encetar algum comportamento que o coloque de facto numa situação de vulnerabilidade e risco face a "B".

A confiança não existe por si numa relação. Desenvolve-se num continuum relacional que depende de múltiplos factores que interagem e se complementam, dando origem a uma relação de confiança. Partindo da discussão da literatura apresentada anteriormente, foi criado um modelo de desenvolvimento da confiança inspirado nos modelos de Dietz e Hartog (2006) e Mayer et al. (1995), que procura integrar todas as condições, factores e processos, que estão associados directa ou indirectamente com o desenvolvimento da confiança (Figura 1).

Assim, "A" formula uma crença sobre a confiabilidade de "B", a partir das suas características, da percepção que tem acerca dos factores de confiabilidade de "B" e das características contextuais (início). A partir desta crença “A” decide se confia ou não em "B", sendo que, a crença e a decisão de confiar são influenciadas por julgamentos racionais, pelas emoções e pelas experiências de relacionamento anteriores (processo). Após a decisão de confiar, "A" enceta um conjunto de comportamentos que o tornam vulnerável a "B" (resultado). O resultado do processo de desenvolvimento da confiança é uma avaliação da congruência entre as expectativas iniciais de " $A$ " e as acções de "B". O feedback resultante deste processo (confirmação ou não das expectativas) vai influenciar as características pessoais de "A", a percepção de "A" sobre a confiabilidade de "B" e a percepção de "A" das características contextuais, no desenvolvimento da relação existente e em próximas relações de confiança.

O modelo de desenvolvimento da confiança aqui apresentado proporcionou a base conceptual para o estudo da confiança entre os residentes e os técnicos das CT's. Ao nível da confiança dos residentes nas Instituições (CT's), não existem modelos estudados que expliquem o seu desenvolvimento. Por essa razão, um dos objectivos deste estudo, como se descreverá no ponto seguinte, é explorar esta área e tentar identificar as bases da confiança dos residentes nestas Instituições. 


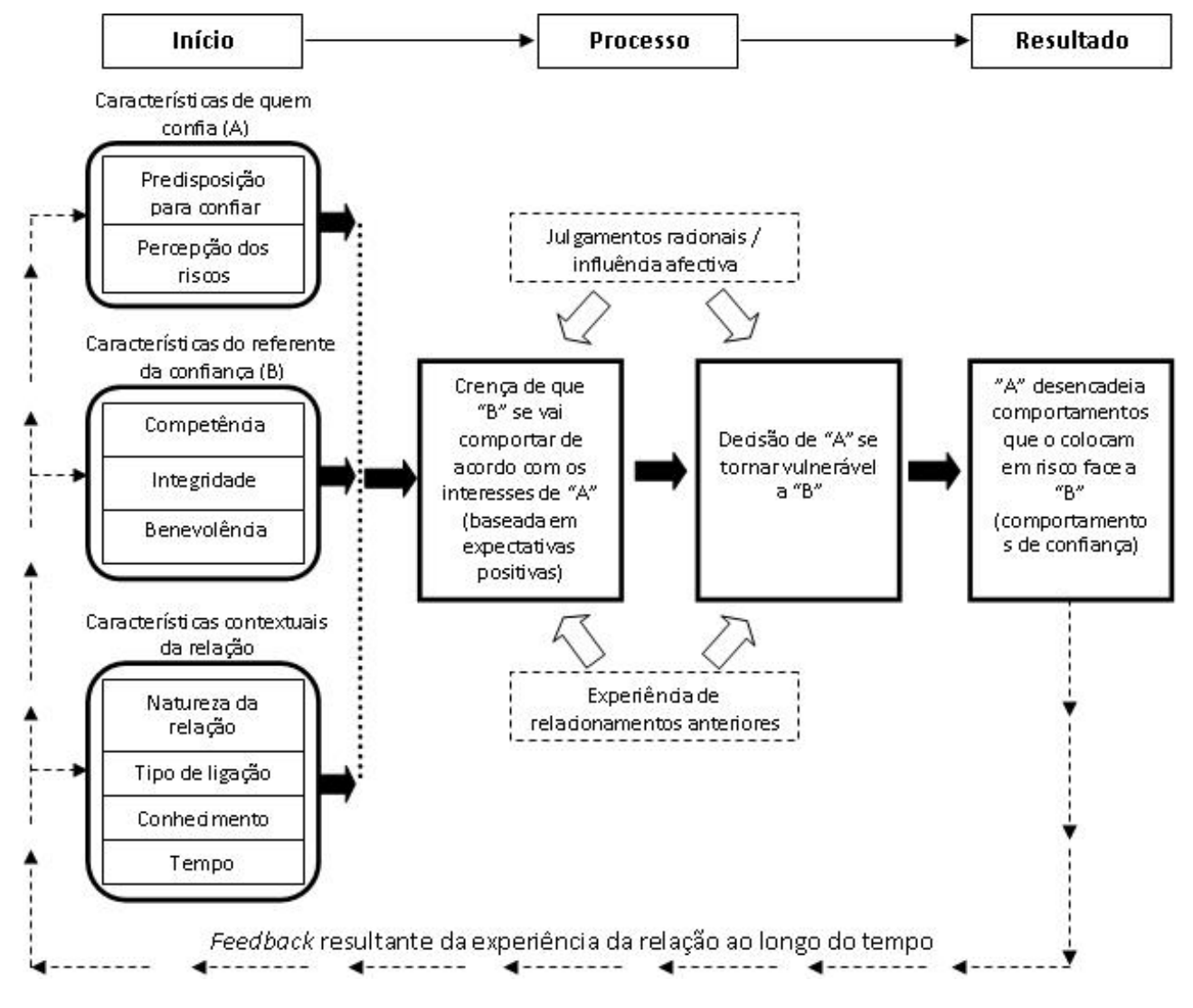

Figura 1. Modelo de Desenvolvimento da Confiança.

\section{Objectivos do Estudo}

Este estudo tem como objectivo aprofundar o conhecimento sobre a confiança no âmbito das CT's, quer ao nível interpessoal na relação entre residentes e técnicos que trabalham nas Instituições, quer no relacionamento dos residentes com as próprias Instituições. Pretende-se compreender o desenvolvimento da confiança, proceder a uma avaliação da mesma e do seu impacto no contexto das CT's.

A primeira questão de investigação deste estudo foi formulada para perceber se o desenvolvimento da confiança é influenciado pelas características pessoais dos residentes, pela percepção dos residentes acerca das características dos técnicos e pelas características do contexto da relação, tal como sugerido no modelo proposto para este estudo.

QI1: Como se desenvolve a confiança dos residentes nos técnicos que trabalham nas CT's?

Foi formulada uma segunda questão de investigação com o objectivo de perceber se existe um nível independente de confiança dos residentes nas CT's enquanto Instituições e como esta se desenvolve.

QI2: Como se desenvolve a confiança dos residentes nas CT's e como esta se diferencia da confiança dos residentes nos técnicos?

Além da exploração dos temas referidos anteriormente, este estudo tem também como objectivo verificar se a confiança dos residentes nos técnicos é moderada pela percepção dos primeiros acerca da "competência”, “integridade” e "benevolência” dos segundos, se existe alguma associação entre a "confiança nos técnicos” e a “confiança nas instituições” e se a confiança é um bom preditor da “adesão ao tratamento”. Para isso, foram criadas as seguintes hipóteses:

H1: O desenvolvimento da confiança dos residentes nos técnicos é moderado pela percepção dos primeiros acerca da “competência”, integridade” e "benevolência” dos segundos.

H2: Os residentes que manifestam níveis mais elevados de "confiança nas CT's” manifestam níveis mais elevados de “confiança nos técnicos”.

H3: A adesão dos residentes das CT's ao tratamento é moderada pelos seus níveis de confiança nos técnicos e nas CT's.

\section{Método}

Face ao carácter inovador e ao elevado nível de complexidade deste estudo decidiu-se recorrer a uma metodologia de investigação mista. Na primeira fase, com o objectivo de responder às duas questões de investigação propostas, o desenho do estudo obedeceu a uma lógica qualitativa, onde a recolha de informação foi realizada através de entrevistas. Para maximizar a fidelidade da informação recolhida optou-se por realizar entrevistas 
com ex residentes e com técnicos que trabalham nas CT's: os ex residentes forneceram informação sobre a experiência pessoal da sua passagem pelas CT's e os técnicos forneceram informação técnica e da sua experiência de trabalho sobre o desenvolvimento da confiança.

A segunda parte do estudo obedeceu a um desenho quantitativo, recorrendo a uma metodologia de recolha de dados através de questionário, preenchido por residentes das CT's. Foi com base nesta segunda fase que se testaram as hipóteses em estudo.

\section{Procedimentos}

As CT's que participaram no estudo foram seleccionadas a partir da lista existente no Instituto da Droga e Toxicodependência, sendo o critério de selecção a sua localização na zona norte de Portugal. A realização das entrevistas e o preenchimento dos questionários ocorreram nas instalações das várias CT’s. A participação foi voluntária e todos os participantes foram informados do objectivo do estudo, da sua natureza confidencial e anónima e instruídos para não referirem o próprio nome nem o de outras pessoas ligadas às CT's. Todas as entrevistas, assim como a passagem dos questionários, foram realizadas pelo mesmo investigador.

\section{Participantes}

Na primeira parte do estudo participaram quatro CT's, das quais foram entrevistados nove técnicos (dois Directores das respectivas CT's; um Psicólogo; um Técnico Superior de Educação; um Assistente Social; um Educador Social e três Monitores) e nove ex residentes que concluíram com sucesso o período de internamento. Para a fase de inquérito participaram nove CT’s, nas quais foram recolhidos 213 questionários, sendo cinco deles considerados inválidos por irregularidades e/ou ausência de respostas a mais de $20 \%$ dos itens. O número de sujeitos por CT varia entre o mínimo de 12 e o máximo de 44 sujeitos. A taxa de resposta aos questionários ficou acima dos 99\%, uma vez que apenas se verificaram duas situações em que os sujeitos não aceitaram participar no estudo. Dos participantes, 14,9\% são do sexo feminino, 19,9\% têm idade compreendida entre os 15 e os 24 anos, 37\% entre os 25 e os 34 anos e 43,3\% têm 35 anos ou mais. Relativamente a experiência de tratamento anteriores, $62,5 \%$ nunca tinha estado anteriormente numa CT, 22,6\% já tinham estado noutras CT’s e 14,9\% dos participantes já tinham estado anteriormente na mesma CT. Quanto ao tempo de permanência nas CT's, 37\% tinham 3 meses ou menos, $18,8 \%$ tinha mais de três meses e menos de seis e $43,8 \%$ seis meses ou mais.

\section{Instrumentos Utilizados}

$\mathrm{Na}$ realização das entrevistas utilizou-se um guião semi-estruturado que permitiu a exploração de temas que, embora não estivessem contemplados à partida, foram surgindo no decorrer das mesmas. Este procedimento decorre da natureza exploratória desta fase do es- tudo e de se ter adoptado a template analysis como metodologia de análise.

O instrumento usado para medir a confiança dos residentes das CT's foi adaptado a partir da versão do questionário sobre confiança organizacional de Mayer e Davis (1999) e Mayer e Gavin (2005), validado para a população por Keating, Silva e Veloso (2010). Este instrumento avalia quatro dimensões: "Competência” (6 itens); "Benevolência / Integridade” (5+5 itens); "Propensão para confiar” (6 itens); “Confiança geral” (10 itens). Os itens são respondidos numa escala tipo Likert de cinco pontos que varia de 1 - discordo fortemente, a 5 - concordo fortemente. A linguagem utilizada nos itens originais foi alterada para proporcionar uma melhor compreensão, tendo em conta as diferenças existentes no contexto deste estudo. Foram acrescentadas duas dimensões ao questionário para medir a “confiança dos residentes nas CT's” (5 itens) e a "adesão ao tratamento” (5 itens). Os itens incluídos nestas duas dimensões foram gerados a partir dos resultados obtidos na template analysis das entrevistas efectuadas (por exemplo: "por aquilo que eu conheço, as pessoas que passam por esta Comunidade Terapêutica conseguem alcançar resultados positivos” - confiança dos residentes nas CT's; “normalmente eu sigo as orientações que me são dadas” - adesão ao tratamento).

\section{Análise dos Dados}

A informação recolhida nas entrevistas foi tratada através de "template analysis" (King, 2008). Na Tabela 1 encontram-se os temas e subtemas que resultaram da codificação inicial dos dados e da estruturação da “template” inicial. Para garantir a qualidade da análise efectuada às entrevistas, dois júris (externos à investigação) procederam a uma avaliação da codificação dos dados e da "template” final. A análise estatística dos dados resultantes dos questionários foi realizada com recurso ao programa informático SPSS ${ }^{\circledR}$ (v.16).

\section{Resultados}

\section{Resultados das Entrevistas}

Para facilitar a compreensão dos dados, as expressões das entrevistas aos técnicos estão sinalizadas com “T” $\mathrm{e}$ aos utentes com "U”, e os números são relativos aos sujeitos entrevistados.

Após a análise dos dados referentes ao desenvolvimento da confiança na relação residente/técnicos, verifica-se que a confiança dos residentes nos técnicos que trabalham nas CT’s é um elemento de base para o estabelecimento da relação terapêutica, quer na perspectiva dos técnicos, quer na perspectiva dos residentes (“. . . à medida que eles vão confiando mais no meu saber, eles começam a colocar-me mais questões e problemas que vivem. Há uma maior procura de ajuda quando aumenta a confiança . . .”. T_2; “. . . se não tivesse confiado nos técnicos e na instituição não teria conseguido levar o tratamento até ao fim”. U_9). 
Tabela 1

Temas e Subtemas que Resultaram da Codificação dos Dados

1. Confiança na relação Residente/Técnicos

1.1. Natureza da Confiança na relação Utente/Técnicos

1.2. Antecedentes da confiança na relação Utente/Técnicos

1.2.1. Competência

1.2.2. Benevolência

1.2.3. Integridade

1.2.4. Propensão para confiar

1.3. Resultados da confiança na relação Utente/Técnicos

\section{Confiança na relação Residente/Instituição}

2.1. Natureza da Confiança na relação Utente/Instituição

2.2. Antecedentes da Confiança na relação Utente/Instituição

2.3. Resultados da confiança na relação Utente/Instituição

\section{Percepção de vulnerabilidade/risco}

3.1. Natureza da vulnerabilidade/ risco

3.2. Implicação da percepção de vulnerabilidade e risco na propensão para confiar

\section{Adesão ao tratamento}

4.1. Comportamentos de adesão

4.2. Comportamentos de não adesão

Entre os factores fundamentais para o desenvolvimento da confiança destacam-se a necessidade de tempo (“. . a ao fim de um mês é que eles estão mais inseridos e começam a sentir-se melhor na CT e a confiar mais um bocadinho. É um processo gradual”. T_1), a existência de conhecimento por parte dos residentes acerca das características dos técnicos (“. . . conhecer as pessoas ajudou-me a confiar nelas”. U_1) e as diferentes características dos residentes que influenciam a sua propensão para confiar ("Alguns têm maior dificuldade em confiar devido ao estilo de vida anterior”. T_2).

Nas características dos técnicos que influenciam a confiança dos residentes encontram-se a benevolência ("O que me fez acreditar na equipa foi a preocupação que eles demonstraram comigo, as vezes que vinham ter comigo, o facto de nunca me terem negado a ajuda". U_3), a integridade ("Se eu achasse que as pessoas não eram muito honestas comigo não confiava neles". U_2) e a competência ("Saber a experiencia, os anos que já trabalhavam, perceber que as pessoas sabiam aquilo que estavam a fazer ajudou-me a acreditar nelas". U_3). No entanto, os dados parecem sugerir que estas características não promovem a confiança, isoladas umas das outras ("Mas mesmo quando reconhecia que os técnicos eram muito competentes, se me sentisse rejeitado por eles não confiava”. U_2)

Outra característica que surge é o contexto ou situação em que o residente se encontra e que o leva a confiar num técnico específico, para resolver problemas específicos ("Eu sabia em que é que cada técnico me podia ajudar. Podia não confiar para umas coisas num técnico e confiar para outras coisas”. U_2).
Os resultados da confiança na relação dos residentes com os técnicos incluem maior predisposição para falar de temas sensíveis para os residentes ("Só uma relação de confiança permite que eles se abram para falar dos temas mais sensíveis”. T_7), níveis mais elevados de participação em todas as actividades, e maior receptividade ("Com a confiança eu fiquei mais disposta a fazer o que me diziam”. U_5), maior predisposição para seguir as orientações dadas pelos técnicos ("Depois de começar a confiar comecei a pôr mais empenho para seguir as orientações”. U_4) e níveis mais elevados de compromisso com as instituições ("Muitas vezes se não fosse essa relação eu tinha desistido do programa". U_3).

A análise dos dados referentes à confiança na relação residente/instituição mostra que os residentes das CT's desenvolvem um sentimento de confiança direccionado à própria instituição e que este é independente da confiança que desenvolvem na relação com os técnicos (“A confiança nos técnicos e na própria instituição teve um papel diferente”. U_9). No início, este tipo de confiança não é tão evidente, mas com o passar do tempo acaba por ganhar maior predominância e por se sobrepor à confiança dos residentes nos técnicos ("À parte de algum técnico que possa ficar mais marcado aquilo que sobressai é a confiança na instituição". T_5). Os factores que influenciam o desenvolvimento da confiança a este nível são os valores e princípios promovidos pelas CT's (“Sem dúvida que o facto de um utente perceber que existe uma estrutura ou uma instituição que tem valores e princípios próprios o ajuda a confiar nas intervenções”. T_3), a evidência de resultados positivos alcançados por antigos residentes ("Começar 
a ver resultados positivos aumentou a minha confiança no programa". U_4), a identificação com o modelo de tratamento adoptado ("Identifiquei-me com a filosofia do método de tratamento e isso ajudou-me a confiar". U_9) e a estrutura que suporta a dinâmica das CT’s (e.g., normas, atribuição de responsabilidades e organização diária):

A confiança que os utentes referem na comunidade assenta no conjunto de tudo que se passa aqui. Desde a equipa técnica, às actividades que fazem aqui, as regras que têm que cumprir. Tem a ver com a própria estrutura da comunidade. (T_6).

Os principais resultados apontados a este nível de confiança são o compromisso de terminar o tratamento (“A confiança na comunidade foi importante para o tratamento ter sucesso". U_9), a intenção de voltar a recorrer aos serviços das CT's caso tenham necessidade de novo tratamento ("Querem voltar para cá porque, apesar de não ter dado resultados positivos, confiam na CT”. T_2), a adesão ao tratamento ("A confiança na instituição teve um peso muito grande para a continuidade no tratamento”. U_3), a intenção de recomendar os serviços das CT's a outras pessoas ("Se encontrar uma pessoa que precise de ajuda eu só me vejo a sugerir esta instituição”. U_3) e maior capacidade para superar problemas decorrentes da perda de confiança nos técnicos ("A confiança que eu tinha na própria instituição compensava a perda de confiança nos técnicos”. U_3).

Tabela 2

Matriz Resultante da AFE

\begin{tabular}{|c|c|c|c|c|c|c|c|}
\hline \multirow{2}{*}{ Itens } & \multicolumn{7}{|c|}{ Componentes } \\
\hline & 1 & 2 & 3 & 4 & 5 & 6 & 7 \\
\hline competencia_2 & 0,819 & 0,141 & 0,170 & 0,167 & 0,059 & 0,136 & $-0,003$ \\
\hline competencia_1 & 0,672 & 0,330 & 0,253 & 0,235 & $-0,030$ & 0,138 & 0,117 \\
\hline competencia_6 & 0,622 & 0,285 & 0,198 & 0,337 & 0,052 & 0,219 & $-0,009$ \\
\hline competencia_5 & 0,621 & 0,393 & 0,169 & 0,309 & 0,156 & 0,178 & 0,027 \\
\hline competencia_3 & 0,613 & 0,409 & 0,244 & 0,286 & 0,120 & 0,145 & 0,093 \\
\hline competencia_4 & 0,592 & 0,404 & 0,187 & 0,333 & 0,173 & 0,178 & 0,098 \\
\hline conf_ct_2 & 0,266 & 0,747 & 0,150 & 0,157 & 0,296 & 0,079 & $-0,051$ \\
\hline conf_ct_1 & 0,180 & 0,739 & 0,161 & 0,222 & 0,279 & 0,136 & $-0,046$ \\
\hline conf_ct_3 & 0,237 & 0,641 & 0,281 & 0,086 & 0,248 & 0,074 & $-0,014$ \\
\hline conf_ct_4 & 0,284 & 0,612 & 0,299 & 0,190 & 0,307 & 0,038 & 0,000 \\
\hline conf_ct_5 & 0,321 & 0,609 & 0,256 & 0,230 & 0,228 & 0,121 & 0,002 \\
\hline integridade_2 & 0,162 & 0,156 & 0,743 & 0,198 & 0,070 & 0,177 & 0,054 \\
\hline integridade_1 & 0,184 & 0,046 & 0,731 & 0,304 & 0,154 & 0,132 & 0,035 \\
\hline integridade_4 & 0,177 & 0,218 & 0,724 & 0,121 & 0,135 & 0,215 & 0,050 \\
\hline integridade_3 & 0,131 & 0,205 & 0,687 & 0,317 & 0,099 & 0,030 & 0,094 \\
\hline integridade_5 & 0,170 & 0,345 & 0,677 & 0,147 & 0,054 & 0,226 & 0,056 \\
\hline benevolencia_3 & 0,092 & 0,192 & 0,121 & 0,785 & 0,103 & 0,068 & 0,023 \\
\hline benevolencia_1 & 0,316 & 0,150 & 0,200 & 0,723 & 0,109 & 0,091 & 0,051 \\
\hline benevolencia_2 & 0,181 & 0,068 & 0,248 & 0,695 & 0,138 & 0,114 & 0,119 \\
\hline benevolencia_5 & 0,259 & 0,193 & 0,317 & 0,682 & 0,117 & 0,076 & 0,081 \\
\hline benevolencia_4 & 0,368 & 0,206 & 0,281 & 0,638 & 0,221 & 0,117 & 0,087 \\
\hline adesao_4 & 0,036 & 0,205 & 0,017 & 0,082 & 0,782 & 0,146 & $-0,005$ \\
\hline adesao_1 & 0,010 & 0,122 & 0,158 & 0,126 & 0,726 & 0,190 & 0,097 \\
\hline adesao_2 & 0,120 & 0,057 & 0,259 & 0,110 & 0,717 & 0,143 & 0,205 \\
\hline adesao_3 & 0,008 & 0,298 & 0,072 & 0,103 & 0,714 & 0,131 & $-0,017$ \\
\hline adesao_5 & 0,200 & 0,345 & $-0,022$ & 0,143 & 0,609 & 0,074 & 0,010 \\
\hline conf_tecnicos_5 & 0,164 & 0,107 & 0,019 & 0,055 & 0,171 & 0,781 & $-0,080$ \\
\hline conf_tecnicos_6 & 0,119 & 0,024 & 0,214 & 0,010 & 0,089 & 0,769 & 0,075 \\
\hline conf_tecnicos_3 & 0,149 & 0,041 & 0,171 & 0,179 & 0,120 & 0,767 & $-0,018$ \\
\hline conf_tecnicos_4 & 0,048 & 0,173 & 0,183 & 0,117 & 0,237 & 0,716 & $-0,059$ \\
\hline propensao_3 & 0,049 & 0,095 & 0,047 & 0,072 & $-0,004$ & $-0,041$ & 0,778 \\
\hline propensao_2 & 0,210 & $-0,029$ & 0,160 & $-0,034$ & 0,140 & $-0,057$ & 0,722 \\
\hline propensao_4 & $-0,245$ & 0,132 & $-0,154$ & 0,193 & $-0,229$ & 0,114 & 0,721 \\
\hline propensao_6 & $-0,094$ & $-0,126$ & 0,102 & $-0,011$ & 0,086 & $-0,006$ & 0,676 \\
\hline propensao_1 & 0,369 & $-0,100$ & $-0,010$ & 0,129 & 0,214 & $-0,048$ & 0,611 \\
\hline
\end{tabular}

Nota. A rotação convergiu em 11 interacções. 


\section{Resultados dos Questionários}

A estrutura relacional das várias dimensões existentes no questionário foi avaliada pela Análise Factorial Exploratória (AFE), com extracção dos factores pelo método dos componentes principais seguido de rotação "varimax", forçando a extraç̧ão de sete factores, de acordo com a matriz conceptual deste estudo (Tabela 2). Tendo-se observado um KMO $=0,923(p<0,001)$ pode-se deduzir que os dados se adequam a este procedimento. A estrutura factorial resultante da AFE explica 67,42\% da variância total dos dados, sendo esta explicada parcialmente pelos factores "competência" $(10,91 \%$ : $\alpha=0,919)$, “confiança nas CT's" $(10,57 \%: \alpha=0,898)$, “integridade” $(10,41 \%$ : $\alpha=0,876)$, "benevolência" $(10,27 \%$ : $\alpha=0,884)$, "adesão ao tratamento" (9,66\%: $\alpha=0,843)$, "confiança nos técnicos” (8,09\%: $\alpha=827)$ e "propensão para confiar” (7,52\%: $\alpha=0,755)$.

Para aferir a independência dos factores e garantir que o método de extraç̧ão forçada não resultou na criação de dimensões que medem o mesmo conceito, foram analisadas as correlações através do coeficiente de correlação de "Spearman" (Tabela 3), que indica alguns coeficientes elevados, mas não o suficiente para indiciar a existência de itens que se confundam ou que meçam os mesmos conceitos.

Tabela 3

Matriz de Correlações dos Factores

\begin{tabular}{|c|c|c|c|c|c|c|c|}
\hline & Competência & Benevolência & Integridade & $\begin{array}{l}\text { Confiança_ } \\
\text { técnicos }\end{array}$ & $\begin{array}{l}\text { Confiança_ } \\
\text { Comunidade }\end{array}$ & $\begin{array}{l}\text { Adesão_ } \\
\text { tratamento }\end{array}$ & Propensão \\
\hline Competência & - & & & & & & \\
\hline Benevolência & $0,679 * * *$ & - & & & & & \\
\hline Integridade & $0,577 * * *$ & $0,565 * * *$ & - & & & & \\
\hline $\begin{array}{l}\text { Confiança nos } \\
\text { técnicos } \\
\text { Confiança na }\end{array}$ & $0,392 * * *$ & $0,350 * * *$ & $0,425^{* * *}$ & - & & & \\
\hline Comunidade & $0,703^{* * *}$ & $0,581 * * *$ & $0,567 * * *$ & $0,377 * * *$ & - & & \\
\hline $\begin{array}{l}\text { Adesão ao } \\
\text { tratamento }\end{array}$ & $0,455^{* * *}$ & $0,396 * * *$ & $0,365 * * *$ & $0,364^{* * *}$ & $0,588 * * *$ & - & \\
\hline Propensão & $0,149 *$ & $0,188 * *$ & 0,122 & 0,018 & 0,036 & 0,125 & - \\
\hline
\end{tabular}

${ }^{*} p<0,05 ;{ }^{* *} p<0,01 ;{ }^{* * *} p<0,001$

Para testar a hipótese 1, foi utilizada a regressão linear múltipla, para obter um modelo que permitisse predizer a variável "confiança nos técnicos", em função das variáveis “competência”, "benevolência” e "integridade”. Este modelo de regressão explica 23,1\% da variância da "confiança nos técnicos” $\left(R_{\mathrm{aj}}^{2}=0,231\right)$. Apesar de, no seu conjunto, este modelo ser significativo $\left(F_{(3,203)}=21,606\right.$, $p<0,001)$, individualmente a variável "benevolência” não teve o mesmo resultado $(t=0,379, p=0,705)$. Assim, este modelo indica que os residentes que percepcionam níveis mais elevados de "competência" e "integridade" manifestam níveis mais elevados de "confiança nos técnicos".

Para testar a hipótese 2 foi utilizado o coeficiente de correlação de "Spearman" (Tabela 3). O resultado mostra uma correlação positiva estatisticamente significativa entre as variáveis "confiança nas CT's" e "confiança nos técnicos" ( $r=0,377, p<0,001)$.

A hipótese 3 foi testada com recurso à análise de regressão linear múltipla. O modelo de regressão testado explica $41 \%$ da variância total da "adesão ao tratamento" e é significativo $\left(R_{\mathrm{aj}}^{2}=0,404\right)\left(F_{(2,202)}=70,159\right.$, $p<0,001)$ e as duas variáveis (confiança nos técnicos" e “confiança na CT”) revelaram ser preditores significativos da "adesão ao tratamento". No entanto, a análise dos coefi-cientes estandardizados "BETA" indica que a variável "confiança nas CT" tem maior influência no aumento da adesão $(\beta=0,536, p<0,001)$, comparativamente com a "confiança nos técnicos" $(\beta=0,207$, $p<0,001)$.

\section{Discussão}

De uma forma geral, os resultados confirmam os pressupostos teóricos subjacentes à criação do modelo de desenvolvimento da confiança, que serviu de base para a investigação. Os resultados das entrevistas sugerem que, de facto, a confiança dos residentes nos técnicos das CT's é influenciada pelas suas características pessoais, pela percepção dos residentes acerca das características da confiabilidade dos técnicos e pelas características do contexto da relação. A excepção a este resultado foi verificada através do modelo de regressão que indica que a "benevolência" não é um preditor significativo da "confiança nos técnicos". Este resultado contraria a tese de Mayer et al. (1995) assumida neste 
estudo, e os resultados da análise das entrevistas. Uma das causas deste "conflito" pode estar na conceptualização dos conceitos. A integridade remete para os valores humanos e princípios morais e a benevolência para a demonstração de boa fé e preocupação genuína pelo outro. Uma análise atenta a estes dois conceitos mostra que, na verdade, os dois podem fazer parte do mesmo conceito, uma vez que a benevolência é também um valor moral. No entanto, a correlação entre ambos é de 0,565, o que não suporta totalmente esta afirmação.

Outra questão explorada neste estudo foi o desenvolvimento de dois níveis de confiança em simultâneo (confiança interpessoal vs confiança organizacional). Os resultados obtidos através da "template analysis” e reforçados pela AFE, através da criação de dois factores independentes, mostram que os residentes desenvolvem um sentimento de confiança nas próprias CT’s, independente da confiança na relação com os técnicos. É de salientar que o desenvolvimento da confiança nas CT's se prolonga mais no tempo e assume características de maior estabilidade, comparativamente com a confiança nos técnicos. As condições que moderam o desenvolvimento da confiança nas CT's são a identificação dos residentes com os valores e princípios promovidos pelas CT's e com o modelo de tratamento adoptado, a evidência de resultados positivos e a crença na eficácia da estrutura das CT’s. A análise das correlações mostra que estes dois níveis de confiança estão associados.

Por fim, confirmaram-se as teorias que defendem que a confiança é um componente fundamental para a adesão ao tratamento. Quer os resultados da “template analysis”, quer o modelo de regressão efectuado para predizer a variável “adesão ao tratamento” em função das variáveis “confiança nos técnicos" e "confiança na CT”, confirmaram este pressuposto. Mesmo sendo a proporção da variabilidade total da variável "adesão ao tratamento" que é explicada pelo modelo de regressão em 41\%, no contexto da adesão ao tratamento nas CT’s, que depende de múltiplos factores não abrangidos por este estudo, este valor torna-se relevante. Neste campo destaca-se a variável “confiança na CT" como o preditor mais forte da variável "adesão ao tratamento”. Embora a influência da confiança nos técnicos e da confiança nas CT’s na adesão ao tratamento tenha alguns pontos comuns, os resultados da "template analysis" mostram um impacto diferente dos dois níveis de confiança. Estes resultados apontam para características de maior estabilidade e solidez ao nível da confiança nas CT’s, comparativamente com a confiança nos técnicos. Enquanto a confiança nos técnicos remete principalmente para resultados a curto prazo (maior participação, maior receptividade, etc.), a confiança nas CT's remete para resultados a longo prazo (compromisso em terminar o tratamento, maior capacidade para superar problemas que decorram na relação com os técnicos, etc.).

\section{Conclusão}

Este trabalho explorou o desenvolvimento da confiança nas CT’s e o seu impacto na adesão ao tratamento. Os principais resultados encontrados foram validados a partir de três fontes de informação independentes: a perspectiva dos técnicos que trabalham nas CT's, dos ex residentes que concluíram com sucesso a passagem pelas CT's e dos residentes em tratamento. Embora estas três fontes de informação não esgotem as possibilidades de conhecimento, elas conferem maior qualidade e fiabilidade aos resultados obtidos.

Além do contributo para o desenvolvimento do estudo da confiança, através da criação de um modelo conceptual que pode servir de base para futuras investigações, este trabalho fornece dados importantes para aqueles que trabalham diariamente nesta área. Tanto os conceitos teóricos como os resultados aqui apresentados podem contribuir para o aperfeiçoamento das CT's, no sentido de maximizar o desenvolvimento de relações de confiança, aumentando a adesão e o sucesso do tratamento. Os resultados apontam para a necessidade das CT's criarem estruturas fortes, assentes em valores e de evidenciarem os casos de sucesso, de forma a fomentarem a confiança dos residentes nas Instituições. De acordo com os resultados obtidos, a necessidade de criar condições para o desenvolvimento da confiança nas CT's acaba por se sobrepor à confiança na relação terapêutica individual.

Foram identificadas duas limitações neste estudo. A primeira limitação foi a falta de um referencial teórico específico sobre o desenvolvimento da confiança nas CT's, que aumentou a complexidade do trabalho desenvolvido. A outra limitação identificada foi a inexistência de uma medida de confiança validada para o contexto das CT's.

Algumas conclusões aqui apresentadas apontam para a necessidade da continuidade de investigação nesta área. Entre elas destacam-se a necessidade de compreender o papel da benevolência no desenvolvimento da confiança dos residentes nos técnicos das CT’s (que neste estudo revelou resultados contraditórios), aprofundar o estudo da confiança dos residentes nas Instituições (importante para a adesão ao tratamento) e a realização de mais estudos para o desenvolvimento de uma medida de confiança adequada ao contexto das CT’s.

\section{Referências}

Bell, D. C. (1994). Connection in therapeutic communities. Substance Use \& Misuse, 29(4), 525-543.

Bigley, A., \& Pearce, J. (1998). Straining for shared meaning in organization science: Problems of trust and distrust. Academy of Management Review, 23(3), 405-421.

Colombrini, M., Lopes, M., \& Figueiredo, R. (2006). Adesão à terapia antiretroviral para HIV/AIDS. Revista da Escola de Enfermagem - USP, 40(4), 576-581. 
Costa, A. C. (2004). Trust. In C. Spielberg (Series Ed.) \& J. M. Prieto (Vol. Ed.), Encyclopedia of Applied Psychology (Vol. 3, pp. 611-620). New York: Elsevier Academic Press.

De Leon, G. (1994). The therapeutic community: Toward a general theory and model. Therapeutic Community: Advances in Research and Application. Research Monograph Series, 144, 21-58.

Dietz, G., \& Hartog, D. (2006). Measuring trust inside organizations. Personnel Review, 35(5), 557-588.

Dugan, E., Trachtenberg, F., \& Hall, M. (2005). Development of abbreviated measures to assess patient trust in a physician, a health insurer, and the medical profession. BMC Health Services Research, 5, 64.

Fontanella, B., \& Turato, E. (2002). Barreiras na relação clínico-paciente em dependentes de substâncias psicoativas procurando tratamento. Revista de Saúde Pública, 36(4), 439-447.

Goudge, J., \& Gilson, L. (2005). How can trust be investigated? Drawing lessons from past experience. Social Science \& Medicine, 61, 1439-1451.

Keating, J., Silva, I., \& Veloso, A. (2010). Teste de um modelo de confiança organizacional. In C. Nogueira, I. Silva, L. Lima, A. T. Almeida, R. Cabecinhas, R. Gomes, et al. (Eds.), Actas do VII Simpósio Nacional de Investigação em Psicologia (pp. 2135-2147). Retrieved from http://www.actassnip2010.com/ conteudos/actas/PsiTrab_10.pdf

King, N. (2008). Template analysis. Retrieved November 12, 2008, from http://www.hud.ac.uk/hhs/research/template_ analysis/whatis.htm

Leite, S., \& Vasconcellos, M. (2003). Adesão à terapêutica medicamentosa: Elementos para a discussão de conceitos e pressupostos adotados na literatura. Ciência \& Saúde Coletiva, 8(3), 775-782.

Lima, H. (2006). Adesão ao tratamento de HIV/AIDS por pacientes com AIDS, tuberculose e usuários de drogas de São Paulo. Tese de Doutorado não-publicada, Faculdade de Saúde Pública, Universidade de São Paulo, SP.

Mayer, R., \& Davis, J. (1999). The effect of the performance appraisal system on trust for management: A field quasiexperiment. Journal of Applied Psychology, 84(1), 123-136.

Mayer, R., Davis, J., \& Schoorman, F. (1995, July/August). An integrative model of organizational trust. Organizational Science, 12(4), 450-467.

Mayer, R., \& Gavin, M. (2005). Trust in management and performance: Who minds the shop while the employees watch the boss? Academy of Management Journal, 48(5), 874-888.

Mcallister, D. (1995). Affect and cognition-based trust as foundations for interpersonal cooperation in organizations. Academy of Management Journal, 38(1), 24-59.

O’Brien, C., \& McLellan, A. (1996). Myths about the treatment of addiction. The Lancet, 347, 237-240.

Ostertag, S., Wright, B., Broadhead, R., \& Altice, L. (2006). Trust and other characteristics associated with health care utilization by injection drug users. Journal of Drug Issues, 36(4), 953-974.

Pickens, R.W., \& Fletcher, B. W. (1991). Overview of treatment issues. National Institute on Drog Abuse. Research Monograph Series, 106, 10-28.

Rosseau, D., Sitkin, S., Burt, R., \& Camerer, C. (1998). Not so different after all: A cross discipline view of trust. Academy of Managment Review, 23(3), 393-404.
Scaduto, A., \& Barbieri, V. (2009). O discurso sobre a adesão de adolescentes ao tratamento da dependência química em uma instituição de saúde pública. Ciência \& Saúde Coletiva, 14(2), 605-614.

Schoorman, F., Mayer, R., \& Davis, J. (2007). An integrative model of organizational trust. Past, present and future. Academy of Management Review, 32(2), 344-354.

Soyez, V., Tatrai, H., Broekaert, E., \& Bracke, R. (2004, August). The implementation of contextual therapy in the therapeutic community for substance abusers: A case study. Journal of Family Therapy, 26(3), 286-305.

Tzafrir, S., \& Dolan, S. (2004). Trust me: A Scale for Measuring Manager-Employee Trust. Management Research, 2(2), 115132.

Vieira, C. (2007). A comunidade terapêutica: Da integração à reinserção. Toxicodependências, 13(3), 15-22.

World Health Organization. (2003). Adherence to long-term therapies: Evidence for action. Retrieved May 5, 2009, from http://whqlibdoc.who.int/publications/2003/9241545992.pdf 